\title{
Applications of B-transform to Some Impulsive Control Problems
}

\author{
Benjamin Oyediran Oyelami \\ Department of Mathematics, Plateau State University, Bokkos, Nigeria
}

Email address:

boyelami2000@yahoo.com

\section{To cite this article:}

Benjamin Oyediran Oyelami. Applications of B-transform to Some Impulsive Control Problems. Mathematics and Computer Science. Vol. 2, No. 1, 2017, pp. 6-13. doi: 10.11648/j.mcs.20170201.12

Received: September 4, 2016; Accepted: January 17, 2017; Published: February 21, 2017

\begin{abstract}
In this paper, B-transform is applied to some impulsive control models and closed solution forms for the models obtained. The problems solved via the B-transform are the third order linear impulsive control systems with bang-bang control, Impulsive delay control systems, Impulsive heat control systems, the Impulsive diffusion problem and the impulsive Gross berg control model. Simulation for the bang bang model show that the solutions are negative and positive in some for given time interval. The solutions also exhibit non-periodic and non-oscillatory behaviour in the given interval. The solutions of impulsive diffusion model possess singularities in given interval of simulation.
\end{abstract}

Keywords: Impulsive, Control Systems, Bang-Bang, B-transform

\section{Introduction}

Impulses are small perturbations acting on a system for short moment of times and can take the form of "jumps", rapid changes in the system or "shocks", short-time mechanical impacts on two or more moving systems etc ([3, 12]).

Many impulsive physical and biological processes occur in nature; the thermionic current in a vacuum tube is not smooth flow of electrons but is subject to fluctuation due to the random emission of electron from the cathode. This phenomenon is called the small-short effect and was discovered by Schotty [7]. This effect can be explained with the help of impulsive theory [9].

Impulsive control systems (ICS) are control systems characterized by short time jumps and shocks that act on the system rapidly. The impulses may act on the state variables describing the systems or on the control variables regulating the systems $([4,6,15,17-18])$.

The solutions of ICS are often discontinuous and are not integrable in the ordinary sense of the word as most hypotheses in the control systems normally assumed. This peculiarity makes ICS not easily accessible to most existing concepts and theorems in the control systems ([9, 16]). Therefore the existing concepts, theories in CS need to be strengthened or new ones developed before applying to the
$\operatorname{ICS}([9])$.

Impulsive control systems are found in biological systems, for examples (See,[4, 9-10, 19]) and in engineering systems (See[9,15-18]) and also have applications in economics wherein the state of an economy of a country can be regulated in a desired way by implementing some policies which has impulsive attributes.

Impulsive control systems are useful in biomedicine where control is strictly needed to regulate biochemical substance in the cells and tissues. ICS also useful in the design of an automatic temperature controlled swimming pool, incubator, nuclear reactors, or heating and cooling system in biological and physical systems that heat or temperature is required to be impulsive [9]. The use of impulsive haematopoiesis control model to measure the replacement of the blood by new blood cells as a result of use of drug, or food supplement and have been obtained together with controllability criteria for the model (See for example [15]).

It is worthy to note that the B-transform was developed by Oyelami and Ale (See [7, 9, 14]) for finding closed solution forms to the fixed moment impulsive systems. B-transform has been applied to solve problems on sickle cell anaemia, HIV/AIDs, fish-hyacinth problem, heart and gas enclosure problems and problem of regulation of cerebrospinal fluid in children with swollen heads (see $[4,7,8,11]$ ). Also related to B-transform is the B-stability which was developed and applied to solve some impulsive control system problems ([4, 
9]).

In the recent times several models are evolving in attempt to some challenging problems in science and technology. Some of these problem are from the control systems that of impulsive family. We will mention in particular solutions of the KdV-Burgers equation, Bagley-Torvik and Painlevé equations [1-2]. Varieties of methods have been developed to find solutions to the above equations. The motivation in this paper is make use of the B-transform method to find closed solution forms to the following problems: bang-bang control, model for a third order linear impulsive control systems, impulsive delay control systems, Impulsive heat control systems, the impulsive diffusion problem and the impulsive Gross berg control model. Moreover, we propose to study the approximate solutions of the impulsive diffusion problem control model.

It is worthy to note that the B -transform has a lot of potential applications unexplored yet. It is, therefore, recommended that more concerted efforts be devoted to the theory.

\section{Preliminary Definitions and Notations}

Throughout the paper we will use of the following notations:

$C\left(\mathfrak{R}^{+}, \mathfrak{R}\right)$ the set of continuous functions defined on $\mathfrak{R}^{+}=[0,+\infty)$ taking values in $\Re$.

$C^{\infty}\left(\mathfrak{R}^{+}\right)$the set of smooth functions defined on $\mathfrak{R}^{+}$.

We consider the impulsive fixed moments $\left\{t_{k}\right\}$, $k=0,1,2, \ldots$ such that $0<t_{0}<t_{1}<t_{2}<\cdots<t_{k}<a, \quad a$ is positive constant.

We define the functions

$$
\begin{aligned}
& f:\left(\begin{array}{ll}
0 & a
\end{array}\right] \times k \rightarrow \Re \\
& g:(0 a] \times k \rightarrow \Re
\end{aligned}
$$

and let $J=(0, a]$ and $J_{i m p}=\left\{t_{k}\right\}_{i=1}^{k}$, we introduce the following piecewise continues function $P C\left(\Re^{+}, \Re\right)$ as follows:

Definition 1:

We say that the function $x: \mathfrak{R}^{+} \rightarrow \mathfrak{R}$ belongs to class $P C\left(\Re^{+}, \mathfrak{R}\right)$ if:

i. The restriction of $x$ to $J /$ Jimp most be continuous function;

ii. There exist two limits such that

$$
\begin{aligned}
& \lim _{\substack{s \rightarrow t_{k} \\
s<t_{k}}} x(s)=x\left(t_{k}^{-}\right) \\
& \lim _{\substack{s \rightarrow t_{k} \\
s>t_{k}}} x(s)=x\left(t_{k}^{+}\right) .
\end{aligned}
$$

The left and right limits of $x(t)$ at $t_{k}$ respectively exist such that $x\left(t_{k}\right)=x\left(t_{k}^{-}\right)$. That is, the function $x(t)$ is left continuous at $t=t_{k}$.

\section{Methods}

\subsection{B -Transform}

B-transform can be apply to a fixed moment impulsive differential equation of the form

$$
\left.\begin{array}{c}
\dot{x}(t)=f(t, x(t)), t \neq t_{k}, k=0,1,2, \ldots \\
\Delta x\left(t_{k}\right)=I\left(x\left(t_{k}\right)\right) \\
0<t_{0}<t_{1}<t_{2}<\ldots<t_{k}, \lim _{k \rightarrow \infty} t_{k}=+\infty
\end{array}\right\}
$$

Where $f$ and $I$ in the eq. (1) are assumed to continuous and satisfy all the conditions the guarantee the existence and the uniqueness of the solution of the eq. (1) (See $[3,9,16]$ ). In [7] we introduced the $B$-transform of a function $x(t)$ with impulses occurring at some fixed moments during the evolutionary process as

$$
B_{n}^{\prime} x(t)=x_{c}(q)+x_{I}(q)
$$

where $x_{c}(q)$ and $x_{I}(q)$ are the components of the $B$ transform and are defined as

$$
x_{c}(q)=L_{c} x(t)=\int_{0}^{\infty} e^{-t / q^{n}} x(t) d t, t \neq t_{k}, k=0,1,2, \ldots
$$

and

$$
x_{I}(q)=L_{I} x(t)=\sum_{t_{0}<t_{k}<t} e^{-t_{k} / q^{n^{\prime}}} I\left(x\left(t_{k}\right)\right)
$$

$n^{\prime}=0,1,2, \ldots$ is the order of the transform. For sake of simplicity, we often choose $n^{\prime}=1$. The advantage of taking $n^{\prime}=1$ lies in the derivation of the inverse transform.

The inverse transforms for components of $x_{c}(q)$ and $x_{I}(q)$ can be obtained as follows:

$$
\begin{gathered}
x_{c}(t)=\int_{v-i \infty}^{v+i \infty} x_{c}(q) e^{s q} d q \\
x(t)=\sum_{t_{o}<t_{k}<t} \psi\left(t_{k}, q\right) I\left(x\left(t_{k}\right)\right) \\
\psi\left(t_{k}, q\right)=\int_{v-i \infty}^{v+i \infty} d q e^{-t_{k} / q^{\prime \prime}+s q} .
\end{gathered}
$$

The $B$-transform is valid in some sets. In [14] symbolic programming method in Maple software was introduced to find the inverse B-transform and the solutions to the impulsive diffusion model and the Von-Foerster Makendrich model were found.

Our first application of B- transform is to find closed solution form to the third order linear impulsive systems with bang-bang control. The bang-bang control models are found 
extensively in many engineering applications. We will make use of the B-transform to find solutions to some classes of the third order linear impulsive control systems with bangbang control.

\subsection{Third Order Linear Impulsive Control Systems}

Consider a third order linear impulsive control system

$$
\begin{gathered}
\cdots(t)+\dot{x}(t)=\mu(t), \quad t \neq t_{k} \\
\Delta x\left(t_{k}\right)=B_{k} x\left(t_{k}\right)
\end{gathered}
$$

For $t, T \in[0,+\infty)$ assuming $\mu(t)$ is bang-bang control such that $|\mu(t)| \leq 1$ for all $t \geq 0$ and $x(T)=\dot{x}(T)=\ddot{x}(T)=0$. $x(0)=x_{0}, \dot{x}(0)=\dot{x}_{0}$ and $\ddot{x}(0)=\ddot{x}_{0}$.

In our next application, we consider the algebraic impulsive delay control systems with impulsive variable being regarded as linear combination of some impulsive variables and the control variable regulating the system contains impulsive delay variables.

\subsection{Impulsive Delay Control System}

Consider an Impulsive delay control system

$$
\begin{aligned}
& \dot{x}(t)=A_{i, j} x(t)+\sum_{i=1}^{n} \sum_{j=1}^{n} B_{i j} x\left(t-c_{i} h_{i}\right)+D u(t), t \neq t_{k}, k=0,1,2, \ldots \\
& \Delta x\left(t=t_{k}\right)=\sum_{k=1}^{N} a_{k} x\left(t_{k}\right) \\
& u(t):=\sum_{k=1}^{N} k_{i} x\left(t-t_{k}\right)
\end{aligned}
$$

For strictly increasing impulsive times $\left\{t_{k}\right\}$ such that

$$
0<t_{0}<t_{1}<t_{2}<\cdots<t_{k}, \lim _{k \rightarrow \infty} t_{k}=+\infty
$$

where $\left(A_{i j}\right)$ and $\left(B_{i j}\right)$ are $n \times n$ constant matrices of dimension $N, x(t) \in R^{N}, t \in \mathfrak{R}^{+}=[0,+\infty) \quad\left(A_{i j}\right)$ is the growth rate matrix for the state vector $x(t) .\left(B_{i j}\right)$, $a_{k}, c_{k}, h_{i}, D$ and $k_{i}$ are some real life parameter describing the impulsive delay control system. The sequence $\left\{B_{i} h_{i}\right\}_{i=1}^{\infty} \subset l^{2}\left(\mathfrak{R}^{+}\right)$is assume to be convergent such that $\left.B_{i} h_{i}\right|_{l^{2}\left(\Re^{+}\right)} \rightarrow B h$ as $i \rightarrow \infty, \quad \theta:=\min _{i, j, k}\left[\left|B_{i} h_{i}\right|,\left|\gamma_{k}\right|,\left|\tau_{k}\right|\right]$, $k_{i}=\left(k_{1}, k_{2}, ?, k_{n}\right)^{T}$.

\subsection{Impulsive Heat Control Systems}

Consider the impulsive heat control systems given by

$$
\left.\begin{array}{l}
\frac{\partial u}{\partial t}=\frac{\partial^{2} u}{\partial x^{2}}+F(t, v), t \geq 0,0 \leq x \leq 1 \\
u\left(t=t_{k}, x\right)=B_{k} u(t, x)=\sum_{k=0}^{N} \beta_{k} \phi\left(t_{k}\right) u\left(t_{k}, x\right)
\end{array}\right\}
$$

Subject to

$$
\left.\begin{array}{l}
u(x, 0)=\phi(x) \\
u(0, t)=a_{0}(t) \\
u(l, t)=a_{1}(t)
\end{array}\right\}
$$

where $u \in P C\left(\Re, \Re^{n}\right) \quad$ the control variable $v, a_{i} \in C\left(\Re^{+}, \mathfrak{R}\right), i=0,1 ; \beta_{k}, \phi \in C^{\infty}\left(\mathfrak{R}^{+}\right)$.

Our next application is impulsive diffusion problem; such problems are extensively found in the molecular biology, neural network. Impulsive diffusion models also have a lot of applications in real life, especially in water and sanitation problems, and in population dynamics ([14]) and complex chemical reaction systems.

\subsection{Impulsive Diffusion Problem}

We consider the application of B-transform to the following impulsive diffusion problem described the following differential equations

$$
\left.\begin{array}{c}
\frac{d u}{d t}=\sum_{k=0}^{N} \sum_{j=0}^{N-k} a_{k j} u_{i}^{k} v_{i}^{k}+\frac{D v}{\delta^{2}} \frac{\partial^{2} u}{\partial x^{2}}+\beta_{1} u, \quad x \neq x_{k}, k=0,1, \cdots \\
\frac{d v}{d t}=\sum_{k=0}^{N} \sum_{j=0}^{N-k} b_{k j} u_{i}^{k} v_{i}^{k}+\frac{D v}{\delta^{2}} \frac{\partial^{2} u}{\partial y^{2}}+\beta_{2} v, \quad y \neq y_{k}, k=0,1,2, \cdots \\
\Delta u\left(t=t_{k}\right)=I_{1}(u)=\sum_{i=1}^{n} a_{i} u_{i}^{k}+b_{k}+f_{1}\left(u_{k}\right) \\
\Delta v\left(t=t_{k}\right)=I_{2}(v)=\sum_{i=1}^{n} b_{i} v_{i}^{k}+c_{k}+f_{2}\left(v_{k}\right)
\end{array}\right\}
$$

Then the impulsive system can be approximated by

$$
\begin{aligned}
\frac{d u_{i}}{d t} & =\sum_{k=0}^{N} \sum_{j=0}^{N-k} a_{k j} u_{i}^{k} v_{i}^{k}+\frac{D v}{\delta^{2}}\left(u_{i-1}-2 u_{i}+u_{i+1}\right)+\beta_{1} u_{i} \\
\frac{d v_{i}}{d t} & =\sum_{k=0}^{N} \sum_{j=0}^{N-k} b_{k j} u_{i}^{k} v_{i}^{k}+\frac{D u}{\delta^{2}}\left(v_{i-1}-2 v_{i}+v_{i+1}\right)+\beta_{2} v_{i} \\
\Delta u_{i} & =I_{1}\left(u_{i}\right)=\sum_{i=1}^{n} a_{i} u_{i}^{k}+b_{k}+f_{1}\left(u_{i}\right) \\
\Delta v_{i} & =I_{2}\left(v_{i}\right)=\sum_{i=1}^{n} b_{i} v_{i}^{k}+c_{k}+f_{2}\left(v_{i}\right)
\end{aligned}
$$

The system in the equation (13) is approximation to the system in the equation (12). It is assumed that the behaviour of the solution to the equation (13) will not be significantly different from the parent equation in the equation (12).

\subsection{Impulsive Grossberg Model}

Most general form of Gross berg model is in perturbed form as follows

$$
\frac{d x_{i}}{d t}=a_{i}\left(x_{i}\right)\left[b_{i}\left(x_{i}\right)-\sum_{j=1}^{N} C_{i j} g_{i}\left(x_{i}\right)\right]+h\left(t, x_{i}, u(t)\right)
$$

where $a_{i}\left(x_{i}\right)$ is the neural output related to internal activity of the neuron; $b\left(x_{i}\right)$ is the neural activity as it jumps from one 
synapse to another; $x_{i}$ is the activity of the neuron and $C_{i j}$ the activity of the synapse being in form of excitatory and inhibitory synapses; $g_{i}\left(x_{i}\right)$ is the neural output. Assume that $\mathrm{a}_{\mathrm{i}}(0)=0, \mathrm{~b}_{\mathrm{i}}(0)=0$ and $\mathrm{g}(0)=0$, and $\mathrm{h}(\mathrm{t}, \mathrm{x}, \mathrm{u}(\mathrm{t}))$ is a non-linear perturbation function with control variable $u(t)$. For insight impulsive analogue of the Grossberg model (See [9])

The matrix $\left(C_{i j}\right)$ and the functions $a_{i}\left(x_{i}\right), b\left(x_{i}\right)$ and $g_{i}\left(x_{i}\right)$ are continuous such that

$$
f\left(t, x_{i}\right)=a\left(x_{i}\right)\left[b_{i}\left(x_{i}\right)-\sum_{j=1}^{N} C_{i j} g_{i}\left(x_{i}\right)\right]+h\left(t, x_{i}, u(t)\right)
$$

If $b_{i}(x)=x_{i+1}-\beta_{i} x_{i}=c=$ constant then the equation becomes neural network describe by an impulsive differential equations of the form:

$$
\begin{aligned}
& \frac{d x_{i}}{d t}=c a_{i}\left(x_{i}\right)-\sum_{j=1}^{N} C_{i j} g_{i}\left(x_{i}\right), t \neq t_{k}, k=0,1,2, \cdots \\
& \left.\Delta x_{i}\right|_{t=t_{k}}=\beta_{i} x_{i}\left(t_{k}\right) \\
& 0<t_{0}<t_{1}<t_{2}<\cdots<t_{k}, \lim _{k \rightarrow \infty} t_{k}=\infty
\end{aligned}
$$

\section{Results and Discussion}

Applying B-transform to the third order linear impulsive control system we have

$$
\left.\begin{array}{l}
L_{C}(\dot{x})=\int_{0}^{\infty} \dot{x} e^{-t / q} d t=x_{0}+\frac{1}{q} x_{C}(q) \\
L_{C}(\ddot{x})=-\ddot{x}_{0}+\frac{1}{q} x_{0}+\frac{1}{q^{2}} x_{C}(q)
\end{array}\right\}
$$

Therefore,

$$
x_{C}(q)=\frac{q^{3}}{q+1}\left[\ddot{x}_{0}+\left(1+\frac{1}{q}\right) \ddot{x}_{0}+\frac{\epsilon_{1}}{q}\right]
$$

Therefore,

$$
\begin{aligned}
x(q) & =x_{C}(q)+x_{I}(q) \\
& =x_{C}(q)+\sum_{t_{0}<t_{k}<t} B_{k} e^{-t_{k} / q} x\left(t_{k}\right) \\
x(q) & =\frac{q^{3}}{1+q}{ }^{\cdots} x_{0}+q^{2} \ddot{x}_{0}+\frac{q^{2}}{1+q} \in_{ \pm 1}+\sum_{t_{0}<t_{k}<1} I\left(x_{k}\right) e^{-t_{k} / q}
\end{aligned}
$$

Hence

$$
x(t)=B^{-1}(x(q))(t)
$$

But

$$
B^{-1}\left(\frac{q^{3}}{1+q}\right)=\int_{C} \frac{q^{3}}{1+q} e^{t q} d q
$$

By residue theory this is equal to

$$
\lim _{q \rightarrow-1}(1+q) \frac{q^{3}}{1+q} e^{t q}=-e^{-t}
$$

In the same vein, using the same theory we have

$$
\left.\begin{array}{l}
B^{-1}\left(q^{2}\right)=\int_{C} q^{3} e^{t q} d q=t \\
B^{-1}\left(\frac{q^{2}}{1+q}\right)=e^{-t}
\end{array}\right)
$$

Therefore,

$$
\left.\begin{array}{l}
x(t)=-e^{-t} \ddot{x}_{0}+t \ddot{x}_{0}+\epsilon_{ \pm} e^{-t}+\sum \phi\left(t_{k}, t\right) I\left(x_{k}\right) \\
\phi\left(t_{k}, t\right)=\int_{C} e^{-t_{k} / q+t q} d q
\end{array}\right\}
$$

We have taken $\epsilon_{ \pm}:= \pm 1$. we simulate the solution to the model using the equation (18) for $\epsilon_{1}=0.02, \dot{u}_{0}=1, \ddot{u_{0}}=0.5, \beta=0.02$ and plotted the graph for $x(t)$ using Maple 17 version and it is the Figure 1 below:

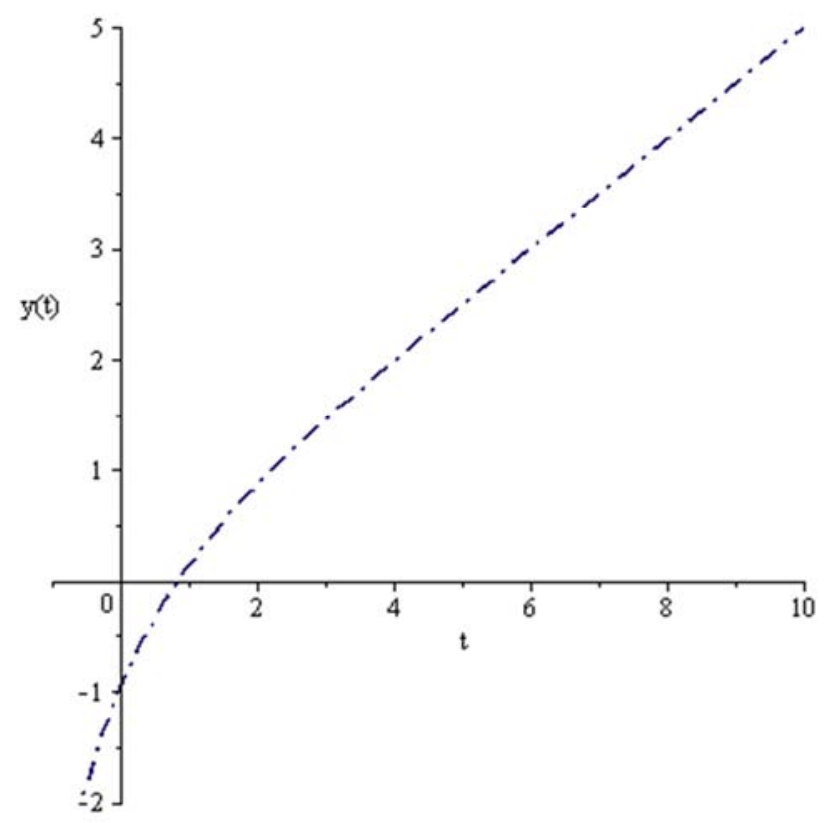

Figure 1. The plot for solution impulsive bang bang control.

In the Figure 1 the solution continue increasing for $t \in \mathfrak{R}$, it is non-oscillatory and no-periodic in the given interval and has zero $x(t)=0$ at $\mathrm{t}=1.5$ seconds. Figure 2 has similar behaviour but zero at about $\mathrm{t}=0.5$ seconds and simulation period in the interval $0 \leq t \leq 50$ seconds. 


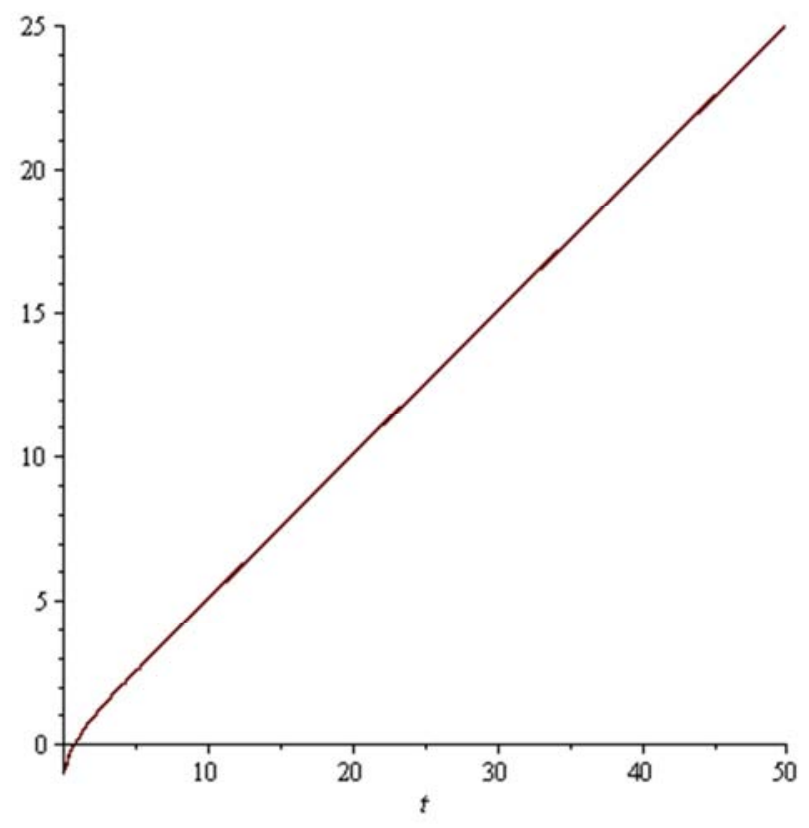

Figure 2. Plot of $x(t), \in_{1}=0.03, \dot{u}_{0}=10, \ddot{u_{0}}=0.05, \beta=0.05$.

Application of B-transform to the impulsive delay control system yields

$$
\begin{aligned}
B_{c}\left(x\left(t-B_{i} h_{i}\right)\right) & =\int_{0}^{\infty} x\left(t-B_{i} h_{i}\right) e^{-t / q} d t \\
& =e^{-B_{i} h_{i} / q}\left[x_{c}(q)+\int_{-B_{i} h_{i}}^{0} x\left(t_{i}\right) e^{t_{i} / q} d t_{i}\right] \\
B_{c} x(t) & =\sum_{j=0}^{r} k_{j} \int_{0}^{\infty} x\left(t-t_{j}\right) e^{-t / q} d t \\
& =\sum_{j=0}^{r} k_{j}\left[e^{-\tau_{j} / q}\left(x_{c}(q)+\int_{-\tau_{0}}^{0} x\left(t_{1}\right) e^{-t_{i} / q} d t_{i}\right)\right] \\
B_{c}(\dot{x}(t)) & =-x_{0}+\frac{1}{q} x(q), \quad B_{c}\left(A_{0} x(t)\right)=A_{0} x(q) .
\end{aligned}
$$

Therefore,

$$
\begin{aligned}
x_{C}(q) & =\left[A_{0}+\left(e^{-B_{i} h_{i} / q}-\frac{1}{q}\right) I\right]^{-1}\left[-x_{0}-e^{-B_{i} h_{i} / q} \int_{-B_{i} h_{i}}^{0} x(s) e^{-s / q} d s\right. \\
& \left.+e^{-t_{0} / q} u(q)+e^{-\tau_{i} / q} \int_{-t_{i}}^{0} u\left(t_{i}\right) e^{-\tau_{1} / q} d t_{i}\right]
\end{aligned}
$$

And

$$
\begin{aligned}
x_{I}(q) & =\sum_{t_{0}<t_{i}<t} I\left(x\left(t_{i}\right)\right) e^{-t_{k} / q} \\
& =\sum_{t_{0}<t_{k}<t 0<\gamma_{k}<t} I\left(x\left(t_{i}\right)\right)=\sum_{0<t_{k}<t 0<\gamma_{k}<t} \sum_{k} e^{t_{k} / q} e^{\gamma_{k} / q} x_{I}(q) .
\end{aligned}
$$

Therefore, $x(q)=x_{C}(q)+x_{I}(q)$ and

$$
x(t)=\frac{1}{2 \pi i} \int_{C} x(q) e^{t q} d q
$$

We observe in the Figure 3 the solutions of the impulsive bang- bang control model for given parameters is negative for $0<t<7.8$ and positive otherwise. The solution is found to be non-oscillatory and non- periodic and the Figure 4 , the solution is negative for $-\infty<t<1.8$ and positive otherwise. The solution also exhibits non-periodic and non-oscillatory behaviour in the given interval.

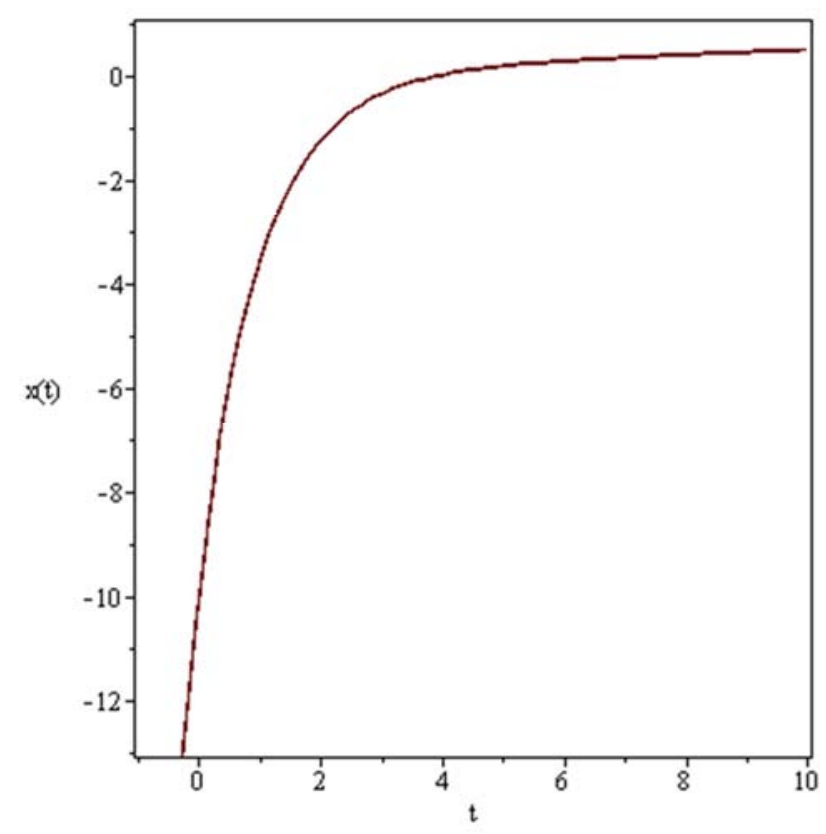

Figure 3. The solution of the impulsive delay control system.

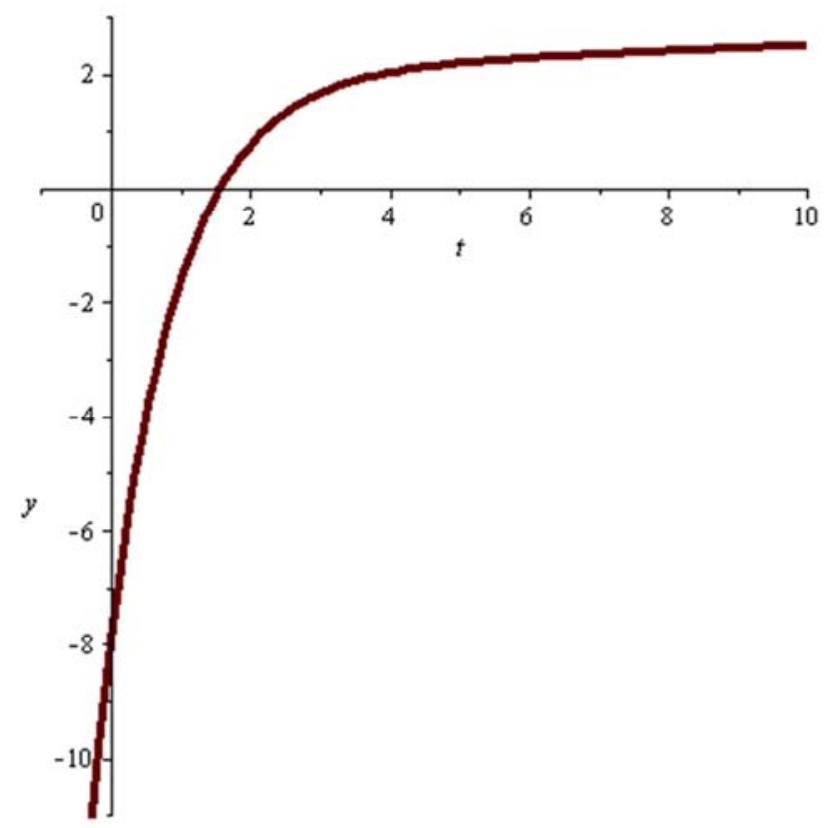

Figure 4. The plot of $x(t), \in_{1}=0.02, \dot{u}_{0}=10, \ddot{u_{0}}=0.05, \beta=0.05, I\left(x_{k}\right)=2^{-k}$.

Application of B-transform to Impulsive heat control systems:

Let $u(t, x)=u(t) u(x)$ Therefore, 


$$
\left.\begin{array}{l}
\frac{\dot{u}(t)}{u(t)}=\frac{u^{\prime}(x)}{u(x)}=-k \\
\Rightarrow u^{\prime}(x)+k u(x)=0 \\
\Delta u\left(t=t_{k}, x\right)=\sum \beta_{k} \phi\left(t_{k}\right) u\left(t_{k}\right) u(x) \\
\Delta u\left(t_{k}\right)=\sum \beta_{k} \phi\left(t_{k}\right) u\left(t_{k}\right)
\end{array}\right\}
$$

Therefore,

$$
u(t)=u_{0} \prod_{t_{0}<t_{k}<t}\left(1+\sum B_{k} \varphi\left(t_{k}\right)\right) e^{-k\left(t-t_{k}\right)}
$$

Application of B-transform to $u^{\prime \prime}(x)$, we set

$$
u(q)=\frac{q^{2}}{k q^{2}-1} \ddot{u}_{0}-\frac{q}{k q^{2}-1} \dot{u}_{0}
$$

Therefore,

$$
u(t)=\frac{1}{2 \pi i} \int_{C} u(q) e^{t q} d q
$$

Where $C$ is a continuous continuing the poles of $u(q)$ which are $q_{+}=\frac{1}{\sqrt{k}}$ and $q_{-}=-\frac{1}{\sqrt{k}}$.

Therefore,

$$
. . u(x)=\frac{1}{2 \sqrt{k}}\left[e^{t / \sqrt{k}}-e^{-t / \sqrt{k}}\right] \ddot{u}_{0}-\frac{1}{2}\left[e^{t / \sqrt{k}}-e^{-t / \sqrt{k}}\right] \dot{u}_{0}
$$

If we take $k=(n \pi)^{2}$. Then,

$$
\begin{aligned}
u_{n}(x) & =\frac{1}{2 n \pi}\left(e^{x / n \pi}-e^{-x / n \pi}\right) \ddot{u}_{0} \\
& -\frac{1}{2}\left(e^{x / n \pi}-e^{-x / n \pi}\right) \ddot{u}_{0} \\
& =\frac{1}{2 n \pi} \sinh \frac{x}{n \pi} \ddot{u}_{0}+\frac{1}{2} \sinh \frac{x}{n \pi} \dot{u}_{0}
\end{aligned}
$$

Therefore

$$
u(x)=\sum_{n=0}^{\infty} u_{n}(x) \sin n \pi x
$$

And

$$
\left.\begin{array}{l}
u(x, t)=u(t) u(x)=u_{0} \Pi_{t_{0}<t_{k}<t}\left(1+\sum_{t_{0}<t_{k}<t} \beta_{k} \phi\left(t_{k}\right)\right) \sum_{n=0}^{\infty} u_{n}(x) e^{-n \pi\left(t-t_{k}\right)} \sin n \pi x \\
u_{n}(x):=\frac{1}{2 \pi n} \sinh \frac{x}{n \pi} \ddot{u}_{0}+\frac{1}{2} \sinh \frac{x}{n \pi} \dot{u}_{0}
\end{array}\right\}
$$

Where $u(x, t)$ is the solution to the impulsive heat model.

We attempted to simulate the solution to the above model, it was found that possess some singularities in the interval $0 \leq t \leq 100$. Applying B-transform to the impulsive diffusion model we have

$$
\begin{aligned}
L_{c}\left(\frac{d u_{i}}{d t}\right) & =-u_{0 i}+\frac{1}{q} \bar{u}_{i c}(q) \\
& =q \sum_{k=0}^{N-k} \sum_{j=0}^{N-k} a_{k j} \bar{u}_{i}^{k} \bar{u}_{i}^{k} \\
& +\frac{D \mu}{\delta^{2}}\left(\bar{u}_{i-1}-2 \bar{u}_{i}+\bar{u}_{i+1}\right)+\beta_{1} \bar{u}_{i} \\
L_{c}\left(\frac{d v_{i}}{d t}\right) & =-v_{0 i}+\frac{1}{q} \bar{v}_{i c}(q) \\
& =q \sum_{k=0}^{N} \sum_{j=0}^{N-k} a_{k j} \bar{u}_{i}^{k} \bar{u}_{i}^{k} \\
& +\frac{D \mu}{\delta^{2}}\left(\bar{v}_{i-1}-2 \bar{v}_{i}+\bar{v}_{i+1}\right)+\beta_{2} \bar{v}_{i} \\
& L_{c}\left(\bar{u}_{i}^{k} \bar{v}_{i}^{k}\right)=\int_{0}^{\infty} u_{i}^{k} v_{i}^{k} e^{-t / q} d t=\frac{1}{q} \int_{0}^{\infty} \int_{0}^{k} u_{i}^{k} v_{i}^{k} e^{-\left(q t+t / q^{2}\right)} d t d t_{1}
\end{aligned}
$$

We note that $\frac{1}{q} \int_{0}^{\infty} e^{-t q} d t=1$.

The system in the equation (12) which was approximate by the system in the equation (13) can be transformed into tridiagonal differential algebraic system of the form:

$$
\begin{gathered}
\dot{X}_{1}=X_{1}^{T} A X_{1}+\alpha A X_{1} \\
\dot{X}_{2}=X_{2}^{T} A X_{2}+\alpha A X_{2} \\
\Delta X_{1}=\alpha X_{1}+b_{1}+f_{1}\left(X_{1}\right) \\
\Delta X_{2}=\alpha X_{2}+b_{1}+f_{2}\left(X_{2}\right)
\end{gathered}
$$

Where $\alpha=\frac{D v}{\delta^{2}}, A_{1}=\operatorname{tridiag}[1,-2,1]=A_{2}$

$$
f_{1}\left(X_{1}\right)=f\left(X_{1}\right), f_{2}\left(X_{2}\right)=f\left(X_{2}\right) .
$$

Apply the B-transform we have

$$
\begin{gathered}
\bar{X}_{1 c}-X_{0}=\bar{X}_{1}^{T} A \bar{X}_{1}+\alpha A_{1} \bar{X}_{1} \\
\bar{X}_{2 c}-X_{0}=\bar{X}_{2}^{T} A \bar{X}_{2}+\alpha A_{2} \bar{X}_{2}
\end{gathered}
$$




$$
\begin{gathered}
\bar{X}_{1 I}=\alpha \bar{X}_{1 I}+\bar{b}_{1}+f_{1}\left(\bar{X}_{1}\right) \\
\bar{X}_{2 I}=\alpha \bar{X}_{2 I}+\bar{b}_{2}+f_{2}\left(\bar{X}_{2}\right) \\
\bar{X}_{1}(q)=\bar{X}_{1 c}+\bar{X}_{1 I}=X_{10}+\bar{X}_{1}^{T} A \bar{X}_{1}+\alpha A_{1} \bar{X}_{1}+\alpha \bar{X}_{1 I}+\bar{b}_{1}+f_{1}\left(\bar{X}_{1}\right) \\
\bar{X}_{2}(q)=\bar{X}_{2 c}+\bar{X}_{2 I}=X_{20}+\bar{X}_{2}^{T} A \bar{X}_{2}+\alpha A_{2} \bar{X}_{2}+\alpha \bar{X}_{2 I}+\bar{b}_{2}+f_{2}\left(\bar{X}_{2}\right)
\end{gathered}
$$

Therefore applying the inverse B-transform we have

$$
\begin{aligned}
& \bar{X}_{i c}(t)=\frac{1}{2 \pi i} \int_{C_{i}} \bar{X}_{i c}(q) e^{t q} d q, i=1,2 . \\
& \bar{X}_{i I}(t)=\frac{1}{2 \pi i} \int_{C_{i}}^{X_{i I}}(q) e^{t q} d q, i=1,2 . \\
& X(t)=\bar{X}_{i c}(t)+\bar{X}_{i I}(t)
\end{aligned}
$$

$C_{i}$ are the contours for which the complex integration for the function $\bar{X}_{i}(q) e^{t q}$ is carried out.

We can use the B-transform to solve the impulsive control Gross berg model,

$$
\begin{aligned}
x_{i}(q)= & x_{c}(q)+x_{I}(q)=q x_{0}+c q a_{i}\left(x_{i}(q)\right)-\sum_{j=1}^{N} C_{i j} g\left(x_{c}(q)\right) \\
& +\sum_{0<t_{0}<t_{k}<t} \mathrm{e}^{-x_{k} / q} I\left(x_{I}\left(t_{k}\right)\right)
\end{aligned}
$$

Applying the inverse B-transform to the we get

$$
\begin{aligned}
x_{i}(t)= & u(t) x_{0}+\frac{1}{2 \pi i} \int_{C} c\left[a_{i}\left(x_{i}(q)\right)-\sum_{j=1}^{N} C_{i j} g\left(x_{c}(q)\right)\right] e^{t q} d q \\
& +\sum_{0<t_{k}<t} \phi\left(\mathrm{x}_{\mathrm{i}}, t_{k}\right) I\left(x\left(t_{k}\right)\right)
\end{aligned}
$$

where

$$
\phi\left(x_{i}, t_{k}\right)=\int_{C} e^{-t_{k} / q+t q} d q
$$

$\mathrm{C}$ is complex domain across which the complex integration is carried out.

For numerical example, take $a_{i}\left(x_{i}(t)\right)=x_{i}(t)$ and $g\left(x_{i}(t)\right)$ then applying the equations $(22) \&(23)$ the solution to the model is $x_{i}(t)=\frac{1}{1-c}\left[u(t) x_{0}-\sum_{i, j}^{n} C_{i j} x_{i}(t)\right], c \neq 1$.

\section{Conclusion}

Impulsive control systems offer many interesting features for modelling several life problems. We considered some few of such real life problems. We only considered an impulsive neural network model; such model has potential applications in telecommunication. The impulsive diffusion model has applications in cellular ecology and population dynamics and the impulsive control systems with a lot of applications in the engineering. The study in this paper should be extended to control systems with impulsive delay functions and more other applications in engineering, science and technology.

\section{References}

[1] A. El-Ajou, O. Abu Arqub, S. Momani, Approximate analytical solution of the nonlinear fractional $\mathrm{KdV}$ Burgers equation: A new iterative algorithm, Journal of Computational Physics 293 (2015) 81-95

[2] Abu Arqub O., B. Maayah, Solutions of Bagley-Torvik and Painlevé equations of fractional order using iterative reproducing kernel algorithm, Neural Computing \& Applications, 2016. DOI 10.1007/s00521-016-2484-4.

[3] Ale S. O. and Oyelami, B. O.: Impulsive System and Applications, Int. J. Math. Edu. Sci. Technol., 2000 Vol., 31, No. 4, 539-544.

[4] Ale S O. and Oyelami B. O. B-Stability and its applications to some constant delay impulsive control models. NMCCOMSATS Proceedings on International Conference on Mathematical Modelling of some Global Challenges in the $21^{\text {st }}$ Century, 2009, pp56-65. http://nmcabuja.org/nmc_proceeding.html.

[5] Bainov, D. D., Lakshikantham, V. and Simeonov, P. S.: Theory of Impulsive Differential Equations (Singapore; World Scientific Publication), 1989.

[6] Hong Shi, Guangming Xie, Controllability and observability criteria for linear piecewise constant impulsive systems, J. Applied Maths. (2012).

[7] Oyelami, B. O. and Ale, S. O.: B-transform method and its applications, in obtaining solutions of some impulsive models. Int. J. Math. Edu. Sc. Tech., 2000, Vol. 31, No. 4, 525-538.

[8] Oyelami, B. O., Ale, S. O., Onumanyi P., Ogidi J. A.: Btransform method and application to sickle cell aneamia. Proc. International Seminar on Theoretical Physics and National Development. Published in a special issue of the African Journal of Physics, 2008, Pp202-220. http://siriusc.ncat.edu/asn/ajp/allissue/ajp-ISOTPAND/index.html

[9] Oyelami B O and Ale S O. Impulsive differential equations and applications to some models: Theory and applications. A monograph, Lambert Academic Publisher Germany, March 24, 2012. ISBN: 978-3-8484-4740-4.

[10] Oyelami, B. O. and Ale, S. O.: B-transform and its applications to a fish-hyacinth model, Int. J. Math. Educ. Sci. Techno 2002 Vol. 33, No. 4, 565 - 573. 
[11] Oyelami, B. O., Ale, S. O., Onumanyi P., Ogidi J. A.: Impulsive HIV-1 model in the presence of antiretroviral drugs using B-transform method. Proc. African Mathematical Union, 2003, 62-76. Oyelami, B. O., Ale, S. O., Onumanyi P.:

[12] Impulsive HIV model using B-transform. Proceedings of National Mathematical Centre on Conference on Computational Mathematics. Vol.2, No.1, 2005, pp50-64. $\mathrm{http}: / /$ nmcabuja.org/nmc_proceedings.html;

http://math.golonka.se/nmc_proceeding.

[13] Oyelami B O and Ale S O. Impulsive model for the absorption of oxygen by the red blood cells in the presence of nitric oxide yielding drugs. African Journal of Physics, Vol.3, 2010. $\mathrm{http}: / /$ sirius-c.ncat.edu/asn/afps/ajp/ajp-

ISOTPAND10/Proc\%20ISPD\%20book145-163.pdf

[14] Oyelami B O and Ale S O. Solutions of Impulsive Diffusion and Von-Foerster Makendrick models using the B-transform. Applied Mathematics Journal, 2013, 4, 1637-1646. (http://www.scirp.org/journal/am) http:/dx.doi.org/10.4236/am.2013.412223.
[15] Oyelami B O. $\delta$-Controllability of impulsive systems and application to some physical and biological control systems. International Journal of Differential Equations and Applications.vol.12, no.3, 2013. http:/dx.doi.org/10.12732/ijdea.v12i3.1093. www.ijpam.eu/en/index.php/idea/article/view/1093/0

[16] Simeonov, P. S. and Bainov, D. D.; Theory of impulsive differential equations: periodic solutions and applications. Longman, Essex, 1993.

[17] Wen C. Y., Li Z. G., Soh Y. C., Analysis and design of impulsive control system, IEEE Transaction on Automatic Control, 46, No. 6 (2001).

[18] Zhi-Hong Guan, J. David Hill, Xuemin (Sherman) Shen, on hybrid impulsive and switching systems and applications to nonlinear control. IEEE Transaction on Automatic Control, 50, No. 7 (2005).pp 1058-1062.

[19] Saker S. H., Alzabut J. O., on the impulsive delay haematopoiesis model with periodic coefficients, Rocky Mountain J. Math., 39, No. 5 (2009), 1657-1688. 